\title{
AN ANALYTICAL COMPARISON OF THE ACOUSTIC ANALOGY AND KIRCHHOFF FORMULATION FOR MOVING SURFACES
}

\author{
Kenneth S. Brentner \\ and \\ F. Farassat \\ Research Engineer \\ Senior Research Scientist \\ NASA Langley Research Center \\ Hampton, Virgina
}

\begin{abstract}
$\underline{\text { Abstract }}$
The Lighthill acoustic analogy, as embodied in the Ffowcs Williams-Hawkings (FW-H) equation, is compared with the Kirchhoff formulation for moving surfaces. A comparison of the two governing equations reveals that the main Kirchhoff advantage (namely nonlinear flow effects are included in the surface integration) is also available to the $\mathrm{FW}-\mathrm{H}$ method if the integration surface used in the $\mathrm{FW}-\mathrm{H}$ equation is not assumed impenetrable. The $\mathrm{FW}-\mathrm{H}$ equation is analytically superior for aeroacoustics because it is based upon the conservation laws of fluid mechanics rather than the wave equation. This means that the $\mathrm{FW}-\mathrm{H}$ equation is valid even if the integration surface is in the nonlinear region. This is demonstrated numerically in the paper. The Kirchhoff approach can lead to substantial errors if the integration surface is not positioned in the linear region. These errors may be hard to identify. Finally, new metrics based on the Sobolev norm are introduced which may be used to compare input data for both quadrupole noise calculations and Kirchhoff noise predictions.
\end{abstract}

\section{Introduction}

A great deal of progress has been made in recent years toward the prediction of rotating-blade noise through methods utilizing first principles. Several reasons account for this progress. First, a detailed and fundamental understanding of how rotor blades generate noise has been gained through several acoustic wind-tunnel and flight tests. Secondly, a rigorous theoretical basis for predicting noise generated by rotating blades has been developed. In fact, several prediction methodologies with a solid physical and mathematical basis are currently available: formulations based upon the Lighthill acoustic analogy ${ }^{1}$ (in particular the Ffowcs Williams and Hawkings $(\mathrm{FW}-\mathrm{H})$ equation $^{2}$ ) and Kirchhoff formulations for both subsonic and supersonic moving surfaces. ${ }^{3,4}$

Presented at the American Helicopter Society 53rd Annual Forum, Virginia Beach, Virginia, April 29 - May 1, 1997.
The $\mathrm{FW}-\mathrm{H}$ equation is an exact rearrangement of the continuity equation and the Navier-Stokes equations into the form of an inhomogenous wave equation with two surface source terms, known as thickness and loading sources, and a volume source term (the quadrupole source from the original Lighthill theory). Although the quadrupole source contribution is insignificant in many subsonic applications, substantially more computational resources are needed for volume integration when the quadrupole source required. The Kirchhoff formulation for moving bodies is also an inhomogenous wave equation with source terms distributed on a surface which encloses all of the physical sources. The Kirchhoff formulation is attractive because no volume integration is necessary.

Although it is useful to have more than one formulation available to predict noise, there is no clear consensus of which to choose for a particular application. A recent numerical comparison by Brentner et al. ${ }^{5}$ of the helicopter rotor noise prediction code WOPWOP,$+{ }^{6-8}$ which uses a $\mathrm{FW}-\mathrm{H}$ based formulation including an approximate quadrupole calculation, with a rotating Kirchhoff code RKIR ${ }^{9,10}$ has shown that both methods can predict the rotor noise equally well. In that work, however, neither method was demonstrated to be clearly superior.

The main purpose of this paper is to analytically compare these two acoustic prediction methodologies and reduce the confusion that currently exists about the relationship between the two methods. This includes a comparison of how the governing equations are derived, highlighting the differences in the derivations. Both analytical comparison and numerical comparisons are necessary to determine whether one method has an advantage in terms of efficiency, accuracy, and robustness over the other. An alternate implementation of the $\mathrm{FW}-\mathrm{H}$ equation is presented which combines the advantages of both the traditional formulation of the FW-H equation and the Kirchhoff formulation. Finally, a useful metric for comparing formulations will be outlined. 


\section{Advantages and Disadvantages}

It is important to briefly consider the advantages and disadvantages of both the FW-H and Kirchhoff formulations at this point in order to understand the motivation for a more in depth analysis.

\section{FW-H Description}

The FW-H approach has several advantages over the Kirchhoff method. First, the three source terms in the $\mathrm{FW}-\mathrm{H}$ equation each have physical meaning which is helpful in understanding the noise generation. The thickness noise is determined completely by the geometry and kinematics of the body. The loading noise is generated by the force acting on the fluid due to the presence of the body. The classification of thickness and loading noise is related to the thickness and loading problems of linearized aerodynamics. Thus, this terminology is consistent with that of aerodynamics. The quadrupole source term accounts for nonlinear effects (i.e., nonlinear wave propagation and steepening, variations in the local sound speed, noise generated by shocks, vorticity, and turbulence in the flow field, etc. $)^{11-13}$

All three source terms are interdependent, yet their physical basis provides information to design quieter rotors. The separation of source terms also is an advantage numerically because not all terms must be computed at all times if it is known that a particular source does not contribute to the sound field (e.g., for low-speed flow the quadrupole may be neglected, in the rotor plane thickness noise is dominant, etc.). A final advantage of $\mathrm{FW}-\mathrm{H}$ based formulations is that these formulations are relatively mature and have robust numerical algorithms. The main disadvantage of the $\mathrm{FW}-\mathrm{H}$ method is that to predict the noise of bodies moving at transonic speeds the quadrupole source must be included. This is a disadvantage because the quadrupole - which is a volume source-ultimately requires a volume integration of the entire source region. Volume integration is computationally expensive and can be difficult to implement. Although the computational effort can be reduced by approximation of the quadrupole, ${ }^{7,8}$ it cannot be avoided completely.

\section{Kirchhoff Description}

The Kirchhoff approach does not suffer from this pitfall because it only has surface source terms. Hence, the Kirchhoff method has been used for the past several years for the prediction of transonic rotor noise. Unlike the FW-H source terms, however, the Kirchhoff source terms are not easily related to thickness, loading, nonlinear effects, or indeed any physical mechanisms. They provide little guidance for design. Another disadvantage of the Kirchhoff method is that the source surface (Kirchhoff surface) must be chosen to be in the linear flow region, such that the input acoustic pressure $p^{\prime} \equiv p-p_{o}$ and its derivatives $\partial p^{\prime} / \partial t$ and $\partial p^{\prime} / \partial n$ are compatible with linear wave propagation. The location of the linear region is not well defined and is problem dependent. It would be desirable to place the Kirchhoff surface well away from the source region, but typically CFD solutions are not as well resolved or as accurate away from the body. Hence, the placement of the Kirchhoff surface is usually a compromise.

\section{Analytical Comparison}

Now that the general characteristics of both the FW-H and Kirchhoff formulations have been described, a more detailed comparison will be helpful. First, we shall consider the development of the governing equations of both approaches to gain insight into the validity of each type of formulation. Then an assessment of an integral formulation for subsonic source motion will be considered.

\section{Governing Equations}

\section{FW-H Equation}

The $\mathrm{FW}-\mathrm{H}$ equation ${ }^{2}$ is the most general form of the Lighthill acoustic analogy and is appropriate for predicting the noise generated by the complex motion of helicopter rotors. The FW-H equation may be derived by embedding the exterior flow problem into a problem in unbounded space by using generalized functions to describe the flow field. To do this, consider a moving surface $f(\mathbf{x}, t)=0$ with a stationary fluid outside. The surface $f=0$ is defined such that $\nabla f=\hat{\mathbf{n}}$, where $\hat{\mathbf{n}}$ is a unit normal vector pointing into the fluid. Inside $f=0$ the generalized flow variables are defined to have their freestream values, i.e.,

$$
\begin{gathered}
\tilde{\rho}=\left\{\begin{array}{cc}
\rho & f>0 \\
\rho_{o} & f<0
\end{array}\right. \\
{\widetilde{\rho u_{i}}}=\left\{\begin{array}{cc}
\rho u_{i} & f>0 \\
0 & f<0
\end{array}\right.
\end{gathered}
$$

and

$$
\tilde{P}_{i j}=\left\{\begin{array}{cc}
P_{i j} & f>0 \\
0 & f<0
\end{array}\right.
$$

where the tilde indicates that the variable is a generalized function defined throughout all space. On the right hand side $\rho, \rho u_{i}$, and $P_{i j}$ are the density, momentum, and compressive stress tensor, respectively. Note that we have absorbed the constant $-p_{o} \delta_{i j}$ into the definition of $P_{i j}$ for convenience, hence, for an inviscid fluid, $P_{i j}=p^{\prime} \delta_{i j}$. Freestream quantities are indicated by the subscript $o$ and $\delta_{i j}$ is the Kronecker delta.

Using definitions (1)-(3), a generalized continuity equation can be written

$$
\frac{\bar{\partial} \tilde{\rho}}{\partial t}+\frac{\bar{\partial} \widetilde{\rho u}_{i}}{\partial x_{i}}=\left(\rho^{\prime} \frac{\partial f}{\partial t}+\rho u_{i} \frac{\partial f}{\partial x_{i}}\right) \delta(f)
$$


where the bar over the derivative operators indicate that generalized differentiation (i.e., differentiation of generalized functions) is implied and $\rho^{\prime} \equiv \rho-\rho_{o}$. Also note that $\partial f / \partial t=-v_{n}, \partial f / \partial x_{i}=\hat{n}_{i}$ and $\delta(f)$ is the Dirac delta function. This generalized continuity equation is valid for the entire space-both inside and outside of the body. The generalized momentum equation can be written

$$
\begin{aligned}
\frac{\bar{\partial} \widetilde{\rho u_{i}}}{\partial t}+ & \frac{\bar{\partial} \rho{\widetilde{u_{i} u_{j}}}_{\partial x_{j}}}{\partial x_{j}} \frac{\bar{\partial} \tilde{P}_{i j}}{\partial x_{j}}= \\
& \left(\rho u_{i} \frac{\partial f}{\partial t}+\left(\rho u_{i} u_{j}+P_{i j}\right) \frac{\partial f}{\partial x_{j}}\right) \delta(f) .
\end{aligned}
$$

Now by taking the time derivative of equation (4) and subtracting the divergence of equation (5), followed with some rearranging, the $\mathrm{FW}-\mathrm{H}$ equation may be written as the following inhomogeneous wave equation:

$$
\begin{aligned}
\square^{2} p^{\prime}(\mathbf{x}, t)= & \frac{\bar{\partial}^{2}}{\partial x_{i} \partial x_{j}}\left[T_{i j} H(f)\right] \\
& -\frac{\partial}{\partial x_{i}}\left[\left(P_{i j} \hat{n}_{j}+\rho u_{i}\left(u_{n}-v_{n}\right)\right) \delta(f)\right] \\
& +\frac{\partial}{\partial t}\left[\left(\rho_{o} v_{n}+\rho\left(u_{n}-v_{n}\right)\right) \delta(f)\right]
\end{aligned}
$$

where $T_{i j}$ is the Lighthill stess tensor, $u_{n}$ is the fluid velocity in the direction normal to the surface $f=0$ and $v_{n}$ is the surface velocity in the direction normal to the surface. On the left hand side we use the customary notation $p^{\prime} \equiv c^{2} \rho^{\prime}$ because the observer location is outside of the source region.

Usually in the derivation of the $\mathrm{FW}-\mathrm{H}$ equation the surface $f=0$ is assumed to be coincident with the physical body surface and impenetrable $\left(u_{n}=v_{n}\right)$. That assumption is not necessary and has not been made in equation (6) so that it may be compared more directly with the governing equation of the Kirchhoff formula for moving surfaces. Ffowcs Williams and Hawkings used slightly different mathematical manipulations, but it is clear from their paper ${ }^{2}$ that they understood it is not essential to choose the integration surface coincidental with the physical body. Recently di Francesantonio ${ }^{14}$ and Pilon and Lyrintzis ${ }^{15}$ have also treated the $\mathrm{FW}-\mathrm{H}$ on a permeable surface, but have used different names to identify the form of the $\mathrm{FW}-\mathrm{H}$ equation given in equation (6). (Pilon and Lyrintzis results appear to be incorrect because they have substitued $p^{\prime}$ for $c^{2} \rho^{\prime}$ in some of their source terms.)

\section{Kirchhoff Equation}

The development of the Kirchhoff formulation, due to Farassat and Myers, ${ }^{3}$ utilizes the same mathematical style and rigor as used in the derivation of the
FW-H equation. The difference is that the domain is now considered in terms of wave propagation. The surface $f=0$ is defined such that all of the acoustic sources are contained inside the surface. Then, the acoustic pressure $p^{\prime}(\mathbf{x}, t)$ is extended such that

$$
\widetilde{p^{\prime}}=\left\{\begin{array}{cc}
p^{\prime} & f>0 \\
0 & f<0
\end{array}\right.
$$

and the generalized wave equation-which is the governing equation for the Kirchhoff formulationbecomes

$$
\begin{aligned}
\square^{2} p^{\prime}(\mathbf{x}, t)= & -\left(\frac{\partial p^{\prime}}{\partial t} \frac{M_{n}}{c}+\frac{\partial p^{\prime}}{\partial n}\right) \delta(f) \\
& -\frac{\partial}{\partial t}\left(p^{\prime} \frac{M_{n}}{c} \delta(f)\right)-\frac{\partial}{\partial x_{i}}\left(p^{\prime} \hat{n}_{i} \delta(f)\right) \\
\equiv & Q_{k i r}
\end{aligned}
$$

where $M_{n}=v_{n} / c$. In this equation is $p^{\prime}$ must be compatible with the wave equation, hence, equation (8) is valid only in the region of the fluid in which the wave equation is the appropriate governing equation.

\section{Source Term Comparison}

It is well known that the wave equation can be derived directly from the conservation laws of fluid mechanics, but it is our objective in this paper to show how equation (8) is related to the $\mathrm{FW}-\mathrm{H}$ equation, equation (6). To that end, we add and subtract terms to the inviscid form of equation (6) to manipulate the source terms into the form of equation (8). This yields

$$
\begin{aligned}
& \square^{2} p^{\prime}(\mathbf{x}, t)=Q_{k i r}+\frac{\bar{\partial}^{2}}{\partial x_{i} \partial x_{j}}\left[T_{i j} H(f)\right] \\
& +\left(\frac{\partial p^{\prime}}{\partial t} \frac{M_{n}}{c}+\frac{\partial p^{\prime}}{\partial n}\right) \delta(f)+\frac{\partial}{\partial t}\left[\left(p^{\prime}-c^{2} \rho^{\prime}\right) \frac{M_{n}}{c} \delta(f)\right] \\
& \quad-\frac{\partial}{\partial x_{i}}\left[\rho u_{i}\left(u_{n}-v_{n}\right) \delta(f)\right]+\frac{\partial}{\partial t}\left[\rho u_{n} \delta(f)\right]
\end{aligned}
$$

If we note that

$$
\frac{\bar{\partial}^{2} H(f)}{\partial t \partial x_{i}}=\frac{\partial}{\partial t}\left(\hat{n}_{i} \delta(f)\right)=-\frac{\partial}{\partial x_{i}}\left(v_{n} \delta(f)\right)
$$

and utilize the continuity and momentum equations we can rewrite equation (9) as

$$
\begin{aligned}
& \square^{2} p^{\prime}(\mathbf{x}, t)=Q_{k i r}+\frac{\bar{\partial}^{2}}{\partial x_{i} \partial x_{j}}\left[T_{i j} H(f)\right] \\
& \quad+\frac{\partial}{\partial t}\left[p^{\prime}-c^{2} \rho^{\prime}\right] \frac{M_{n}}{c} \delta(f)+\frac{\partial}{\partial t}\left[\left(p^{\prime}-c^{2} \rho^{\prime}\right) \frac{M_{n}}{c} \delta(f)\right] \\
& \quad-\frac{\partial}{\partial x_{j}}\left[\rho u_{i} u_{j}\right] \hat{n}_{i} \delta(f)-\frac{\partial}{\partial x_{i}}\left[\rho u_{i} u_{n} \delta(f)\right] .
\end{aligned}
$$

This form of the FW-H equation is helpful because the source terms that are not found in the Kirchhoff 
governing equation are easily identified. This is an important result of this paper. All of the additional source terms are second order and may be neglected in the linear flow region. This was precisely Lighthill's original premise - the wave equation is the appropriate governing equation outside of a limited source region. In fact, when $p^{\prime}=c^{2} \rho^{\prime}$ equation (11) becomes

$$
\square^{2} p^{\prime}(\mathbf{x}, t)=Q_{k i r}+\frac{\bar{\partial}^{2} \rho u_{i} u_{j}}{\partial x_{i} \partial x_{j}} H(f) .
$$

Notice that the Heaviside function has been taken out of the equation (11) quadrupole source term in the manipulations leading to equation (12). The only source term remaining which is not in equation (8) is clearly second order in the perturbation quantity $u_{i}$. This term would be neglected in the derivation of the wave equation from the fluid conservation laws. Hence, we have shown that the FW-H and Kirchhoff formulations are indeed equivalent when the integration surface for both is placed in the linear region of the flow (i.e., where the input data is compatible with the wave equation).

The FW-H equation and the Kirchhoff are quite different, however, when the integration surface is in the source region. The implications of this difference is demonstrated later with numerical examples. If the $\mathrm{FW}-\mathrm{H}$ equation integration surface is on the body or in the source region, the quadrupole-a volume source term-must be included to accurately predict the noise. Therefore, we can infer that as we move the integration surface of the $\mathrm{FW}-\mathrm{H}$ equation away from the body, the contribution of the volume quadrupole contained within the surface must now be accounted for by the surface source terms. We shall numerically demonstrate this later.

For completeness, equation (11) can be simplified by canceling terms and rearranging. The result is

$$
\begin{aligned}
& \square^{2} p^{\prime}(\mathbf{x}, t)=-\left(\frac{\partial c^{2} \rho^{\prime}}{\partial t} \frac{M_{n}}{c}+\frac{\partial \rho u_{i}}{\partial t} \hat{n}_{i}\right) \delta(f) \\
& \quad-\frac{\partial}{\partial t}\left(c^{2} \rho^{\prime} \frac{M_{n}}{c} \delta(f)\right)-\frac{\partial}{\partial x_{i}}\left[\left(p^{\prime} \hat{n}_{i}+\rho u_{i} u_{n}\right) \delta(f)\right] \\
& \quad+\frac{\partial^{2}}{\partial x_{i} \partial x_{j}}\left[T_{i j} H(f)\right] .
\end{aligned}
$$

Notice that the surface source terms in equation (13) are closely related to equation (8). In fact by substituting $c^{2} \rho^{\prime}$ for $p^{\prime}$ in the time derivative terms in equation (8) and $\rho u_{i} u_{j}+p^{\prime} \delta_{i j}$ in the spatial derivative terms we can get the surface source terms in equation (13). (The momentum equation was used to exchange $\partial\left(\rho u_{i} u_{j}+p^{\prime} \delta_{i j}\right) / \partial x_{j}$ with $-\partial \rho u_{i} / \partial t$ in equation (13).) While the correspondence between equation (13) and equation (8) is interesting, equation (13) has two pitfalls: it is not easily recognized as the FW-H equation, and there are no clear connections between the form of the source terms and the problem physics.

\section{An Integral Formulation}

Now that the relationship between the FW-H equation and the Kirchhoff formulation has been developed on the governing equation level, we would like to develop an applicable integral form which is appropriate for subsonic source motion. This is needed for ultimate implementation and numerical comparison of the different formulations.

A slightly modified integral formulation for the FW-H equation is needed because the current practice is to assume that the $\mathrm{FW}-\mathrm{H}$ integration surface corresponds to the body and is impenetrable. Equation (6) is the appropriate form of the FW-H equation to start the development of an integral representation which has the same form as the traditional application of the FW-H equation. Following di Francesantonio, ${ }^{14}$ we define new variables $U_{i}$ and $L_{i}$ as

$$
U_{i}=\left(1-\frac{\rho}{\rho_{o}}\right) v_{i}+\frac{\rho u_{i}}{\rho_{o}}
$$

and

$$
L_{i}=P_{i j} \hat{n}_{j}+\rho u_{i}\left(u_{n}-v_{n}\right) .
$$

We have chosen a slightly different, but equivalent, definitions from that of reference 14 because $\rho$ and $\rho u_{i}$ are conservation variables often utilized in CFD codes. With these definitions, the FW-H equation may be written in its standard differential form:

$$
\begin{aligned}
& \square^{2} p^{\prime}(\mathbf{x}, t)=\frac{\partial^{2}}{\partial x_{i} \partial x_{j}}\left[T_{i j} H(f)\right] \\
& -\frac{\partial}{\partial x_{i}}\left[L_{i} \delta(f)\right]+\frac{\partial}{\partial t}\left[\left(\rho_{o} U_{n}\right) \delta(f)\right] .
\end{aligned}
$$

This equation is particularly useful because Farassat's formulation $1 \mathrm{~A}^{6,16}$ can be utilized directly to write an integral representation of the solution as

$$
p^{\prime}(\mathbf{x}, t)=p_{T}^{\prime}(\mathbf{x}, t)+p_{L}^{\prime}(\mathbf{x}, t)+p_{Q}^{\prime}(\mathbf{x}, t)
$$

where

$$
\begin{aligned}
4 \pi p_{T}^{\prime}(\mathbf{x}, t) & =\int_{f=0}\left[\frac{\rho_{o}\left(\dot{U}_{n}+U_{\dot{n}}\right)}{r\left(1-M_{r}\right)^{2}}\right]_{r e t} d S \\
+ & \int_{f=0}\left[\frac{\rho_{o} U_{n}\left(r \dot{M}_{r}+c\left(M_{r}-M^{2}\right)\right)}{r^{2}\left(1-M_{r}\right)^{3}}\right]_{r e t} d S,
\end{aligned}
$$




$$
\begin{aligned}
4 \pi p_{L}^{\prime}(\mathbf{x}, t) & =\frac{1}{c} \int_{f=0}\left[\frac{\dot{L}_{r}}{r\left(1-M_{r}\right)^{2}}\right]_{r e t} d S \\
+ & \int_{f=0}\left[\frac{L_{r}-L_{M}}{r^{2}\left(1-M_{r}\right)^{2}}\right]_{r e t} d S \\
+ & \frac{1}{c} \int_{f=0}\left[\frac{L_{r}\left(r \dot{M}_{r}+c\left(M_{r}-M^{2}\right)\right)}{r^{2}\left(1-M_{r}\right)^{3}}\right]_{r e t} d S,
\end{aligned}
$$

and $p_{Q}^{\prime}(\mathbf{x}, t)$ can be determined by any method currently available (e.g., see reference 8 ). In equation (17) the dot over a variable implies source time differentiation of that variable, $L_{M}=L_{i} M_{i}$, and a subscript $r$ or $n$ indicates a dot product of the vector with the unit vector in the radiation direction $\hat{\mathbf{r}}$ or the unit vector in the surface normal direction $\hat{\mathbf{n}}$, respectively.

Current rotor noise prediction codes can easily be modified to accommodate this new implementation of the $\mathrm{FW}-\mathrm{H}$ equation. The major difference is that the integration surface is no longer restricted to the rotor blade surface and in addition to $p^{\prime}$, the values of $\rho$, $\rho u_{i}$ are needed as input. When the surface does correspond to the blade surface, the separation of source terms into thickness, loading, and quadrupole noise still has physical meaning; otherwise, the separation of the source terms into $p_{T}^{\prime}, p_{L}^{\prime}$, and $p_{Q}^{\prime}$ is only mathematical. Hence, the ability to give physical interpretation to the source terms continues to be a distinct and unique advantage of the $\mathrm{FW}-\mathrm{H}$ equation.

\section{Numerical Comparison of Formulations}

Although we have shown analytically that the $\mathrm{FW}-\mathrm{H}$ formulation has advantages over the Kirchhoff formulation, what really matters is how they compare in practice. Some comparisons have already been made (e.g., see references 5,14 and 17). In reference 14, di Francesantonio concluded that the main advantage of the FW-H equation applied on a Kirchhoff-type integration surface is that interaction with CFD codes is easier because the normal derivative of pressure is no longer required. If this is the only advantage, and indeed we recognize that the normal derivative calculation can be cumbersome, a simple solution would be to make the substitution

$$
\frac{\partial p}{\partial n}=-\hat{n}_{i} \frac{\partial \rho u_{i}}{\partial t}
$$

in equation (8). This result is just the linear momentum equation, which is applicable in the linear flow region. Nevertheless, we believe there are other advantages which we will now demonstrate numerically.

For this work, a new computer code based on a modification of the RKIR code (Rotating KIRchhoff formulation) developed by Lyrintzis et al. ${ }^{10}$ has been de- veloped to test the numerical implementation of equation (17) without the quadrupole source term. The modified code is called FW-H/RKIR in this paper. RKIR was chosen as the platform to test the new FW-H implementation primarily because it already performs integration on a surface some distance from a rotor blade and has been coupled to the full potential flow solver FPRBVI. ${ }^{18,19}$ A third code, WOP$\mathrm{WOP}+{ }^{8}$ which utilizes the traditional $\mathrm{FW}-\mathrm{H}$ implementation (surface integration on the blade surface and an approximate quadrupole implementation) will also be used in the comparison.

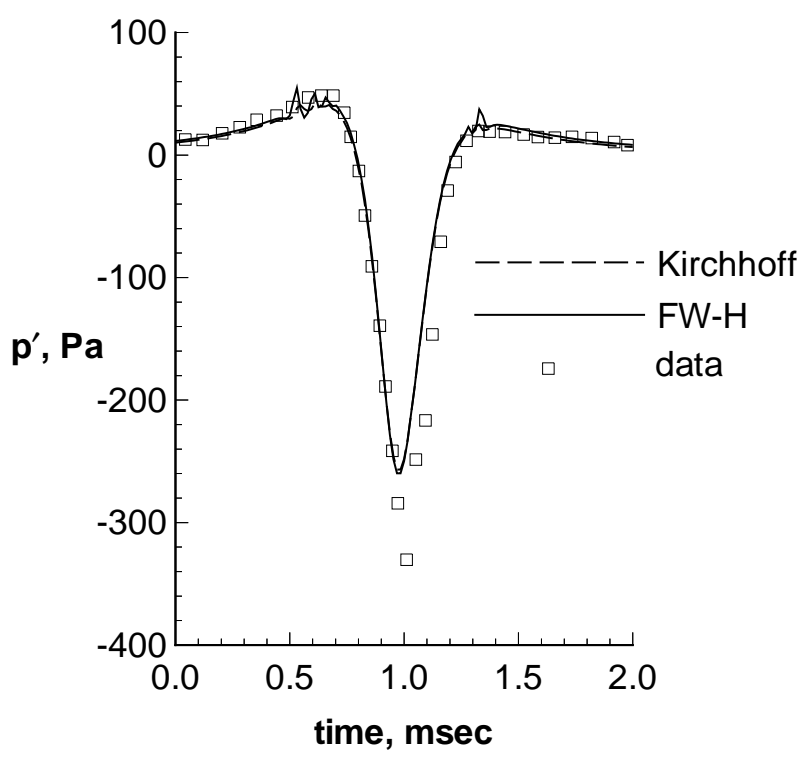

Figure 1. Comparison of predicted and measured ${ }^{20}$ acoustic pressure at an in-plane observer location, $3.4 R$ from the rotor hub of an untwisted UH-1H model rotor in hover $\left(M_{H}=0.88\right)$.

The first comparison is for an untwisted UH-1H model-scale rotor operating in hover with a hover-tip Mach number $M_{H}=0.88 .^{20}$ Figure 1 shows a comparison of acoustic pressure time history for both the Kirchhoff and $\mathrm{FW}-\mathrm{H}$ methods on a integration surface which was located approximately 1.37 chords away from the rotor in the direction normal to the blade surface and extending 1.25 chords beyond the blade tip. The full potential computation was performed on a $80 \times 36 \times 24$ grid, which is somewhat coarse. The two computations are almost indistinguishable in this case - an indication that the integration surface is indeed in the linear flow region. The underprediction of the negative peak is a result of using a coarse grid. Brentner et al. ${ }^{5}$ found that the agreement is improved with a finer grid. Small oscillations in the signal, near 


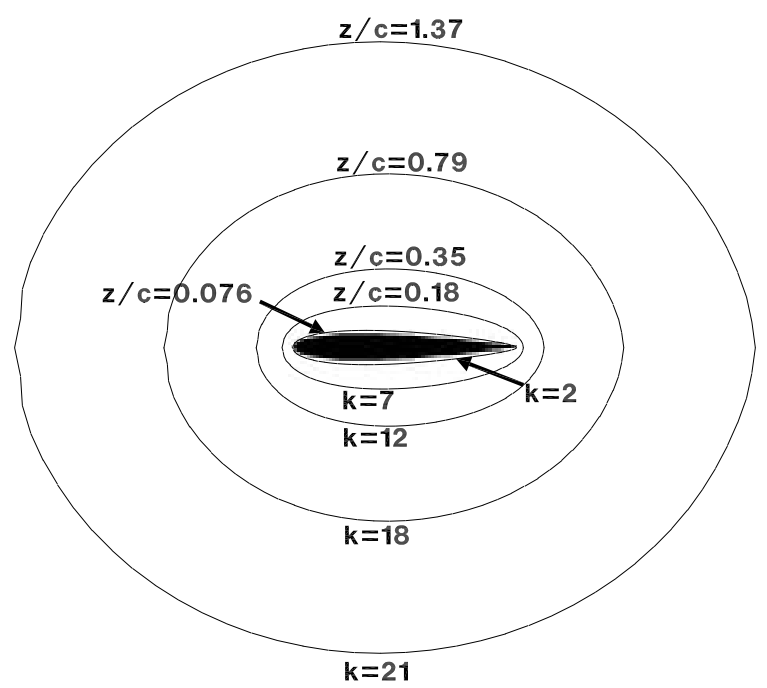

Figure 2. Cross section showing the location of the integration surfaces with respect to the rotor blade. The vertical distance from the blade chord, in units of chord length, are labeled $z / c$. The value of the grid index normal to the blade is labeled $k$.

the two positive peaks, are evident in both the Kirchhoff and FW-H solutions. These oscillations are almost certainly due to inaccurate quadrature over panels moving at high speed. The oscillations disappear as the integration surface size is reduced.

Now that the FW-H/RKIR code has been introduced, we wish to examine the sensitivity of each formulation to the placement of the integration surface. Brentner et al. ${ }^{5}$ found that the Kirchhoff solution varied somewhat with location of the integration surface. Figure 2 shows a cross section of five different integration (Kirchhoff) surface locations ranging from one grid line off the surface to 1.37 chordlengths off the surface. The Kirchhoff acoustic pressure predictions from RKIR code for each of these surface locations are shown in figure 3. As the integration surface is brought nearer to the surface and the input data is no longer compatible with the linear wave propagation assumption, the predicted acoustic pressure becomes meaningless. Although expected, this aspect of the Kirchhoff method is troublesome. If the surface is not positioned properly the error can be substantial. Worse yet, if the integration surface is just positioned slightly in the nonlinear region the solution may be significantly in error but not enough so to be easily recognized.

Figure 4 shows the noise prediction using the $\mathrm{FW}-\mathrm{H}$

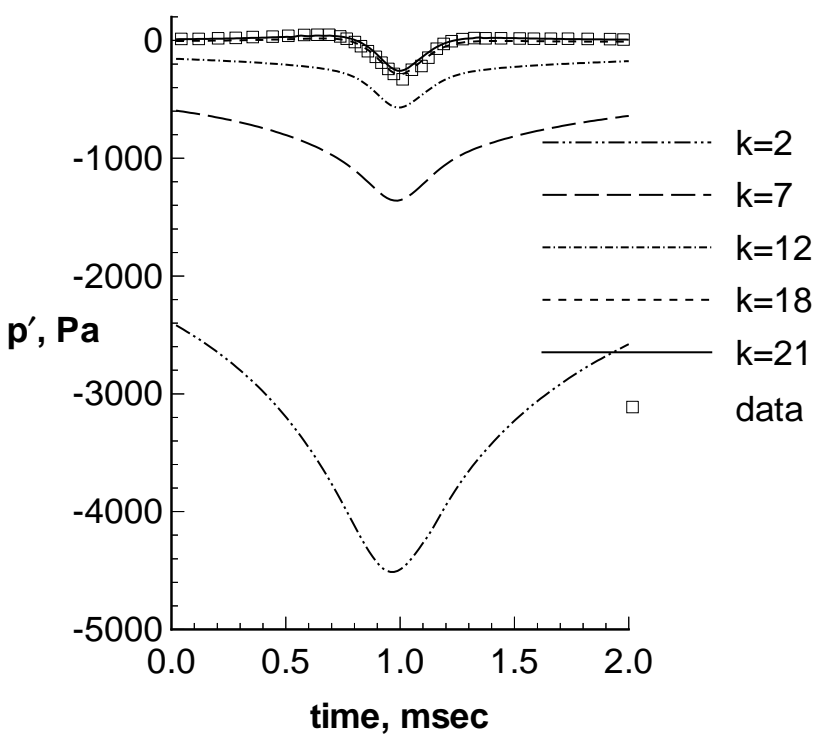

Figure 3. Comparison of predicted acoustic pressure using the Kirchhoff formulation with varying integration surface locations. These predictions are for an observer located $3.4 R$ from a UH-1H model rotor hovering at $M_{H}=0.88$. The experimental data is from reference 20 .

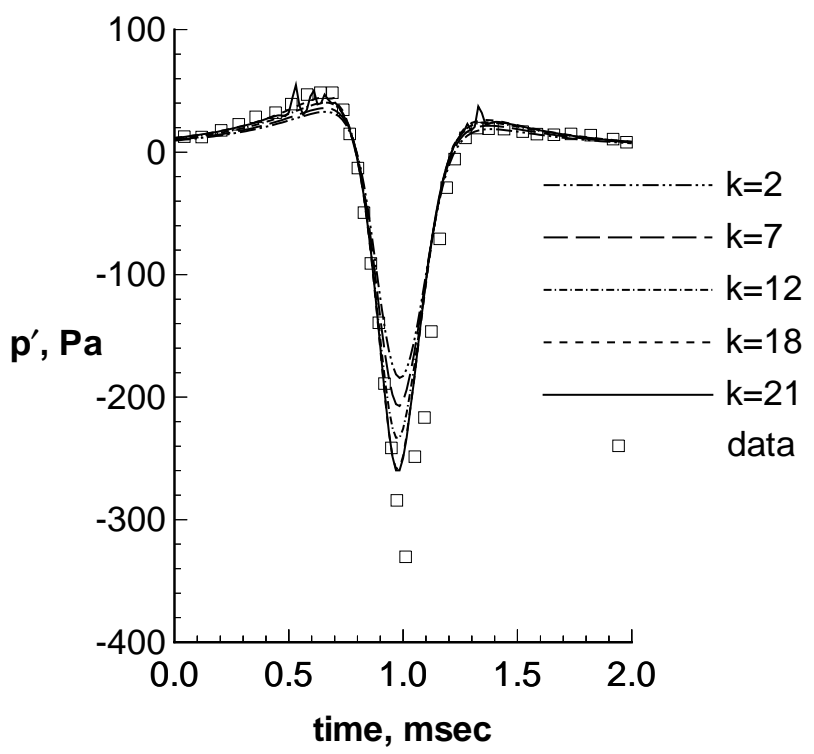

Figure 4. Comparison of predicted acoustic pressure using the $\mathrm{FW}-\mathrm{H}$ formulation integration surface locations. These predictions are for an observer located $3.4 R$ from a $\mathrm{UH}-1 \mathrm{H}$ model rotor hovering at $M_{H}=0.88$. The experimental data is from reference 20. 


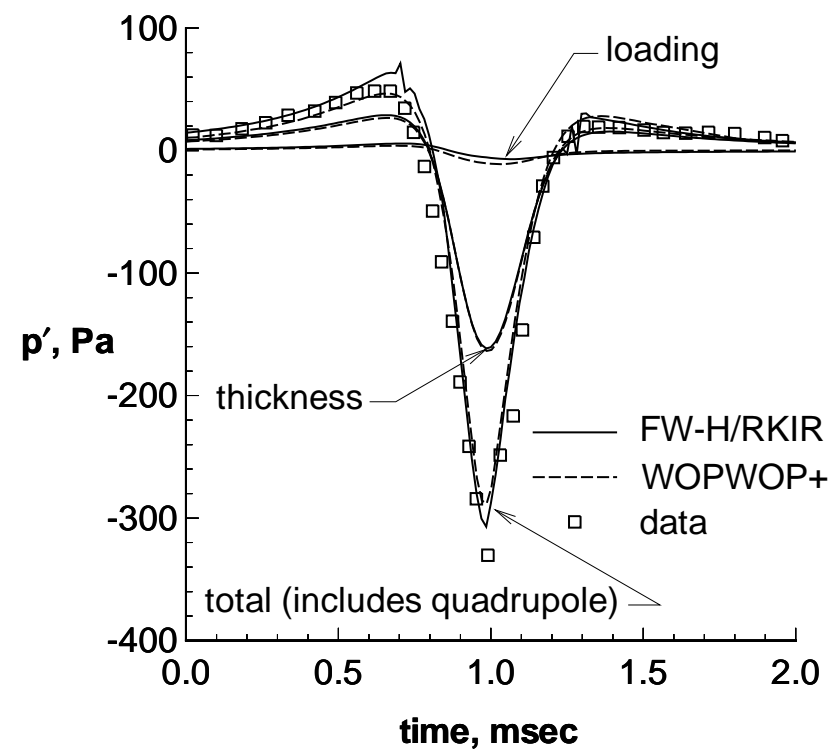

Figure 5. Comparison of noise components predicted by the FW-H/RKIR and WOPWOP + codes for a hover UH-1H model rotor $\left(M_{H}=0.88\right.$, inplane observer $3.4 R$ from rotor hub).

formulation given in equation (17) for the same set of integration surfaces and CFD input data as shown in figure 3 . The volume quadrupole source, which exists only outside the integration surface, has been neglected in this calculation. The advantage of the FW-H formulation is clear: for an integration surface near or on the physical body, the predicted acoustic signal is essentially that of thickness and loading noise alone. As the integration surface is moved farther and farther away, more and more of the quadrupole source contribution is accounted for by the surface integrals. Hence, we would say that the principal advantage of the $\mathrm{FW}-\mathrm{H}$ formulation for aeroacoustics is the relaxation of integration surface placement restrictions. In fact when the volume quadrupole source is included in the noise computation, the location of the integration surface is only a matter of choice and convenience.

Another traditional advantage of the $\mathrm{FW}-\mathrm{H}$ method is the physical basis and identification of the source terms. If equation (17) is used on a surface away from the body this feature is not retained, however, a second computation can be made on the body surface to determine thickness and loading noise. This has been done in figure 5, which is a comparison of $\mathrm{FW}-\mathrm{H} / \mathrm{RKIR}$ predictions with a WOPWOP + prediction. Two FW$\mathrm{H} / \mathrm{RKIR}$ computations are show in figure 5: an integration surface coincident with the rotor blade surface to predict thickness and loading noise, and an inte-

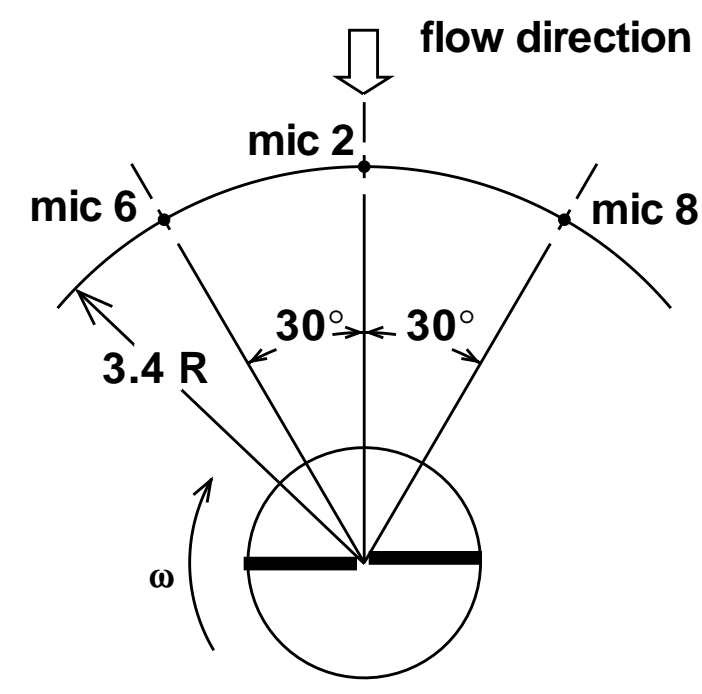

Figure 6. Schematic showing three inplane microphone locations used in the the measurement of noise from the model scale Operational Loads Survey (OLS) rotor. ${ }^{21}$

gration surface located approximately 1.5 chordlengths away from the blade to predict the total noise. Note that the thickness noise predictions from WOPWOP+ and $\mathrm{FW}-\mathrm{H} / \mathrm{RKIR}$ are identical and there is only a small difference in the predicted loading noise. The difference in the predicted loading noise is due to a difference in how the integration over the blade tip face is handled. The total noise, which includes the effect of the quadrupole, is also in very close agreement even though the volume used in WOPWOP + is not identical to the region enclosed in the $\mathrm{FW}-\mathrm{H} / \mathrm{RKIR}$ surface integration. The negative peak is also in better agreement than the earlier figures because an Euler solution from Baeder ${ }^{17}$ was used as input rather than the FPRBVI solution used in figures 1, 3, and 4 .

A model-scale test of the Operational Loads Survey (OLS) rotor is selected for a final comparison. The predicted noise from $\mathrm{FW}-\mathrm{H} / \mathrm{RKIR}$, RKIR, and WOPWOP + are compared with experimental data ${ }^{21}$ at three inplane microphone positions, shown schematically in figure 6 . The rotor was operating in a forward flight condition with advancing-tip Mach number $M_{A T}=0.84$ and advance ratio $\mu=0.27$. A FPRBVI solution $(80 \times 36 \times 24$ grid $)$ was used as input data for all three noise predictions shown in figure 7 . All of these predictions agree quite well with the databoth in directivity and amplitude. All of the codes underpredict the negative peak pressure for microphone 6 , but this is most likely attributed to the FPRBVI solution rather that the noise prediction codes. The 


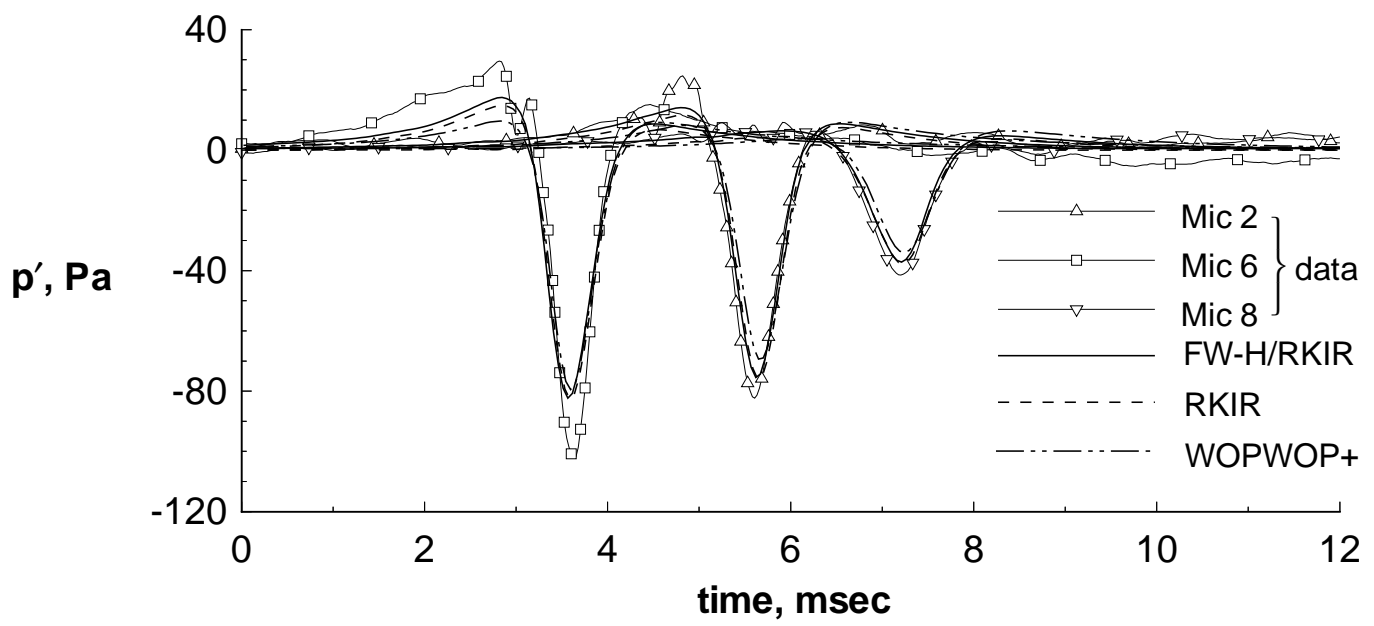

Figure 7. Comparison of predicted and measured ${ }^{21}$ acoustic pressure at three microphone locations for the model scale Operation Loads Survey (OLS) rotor $\left(M_{A T}=0.84, \mu=0.27\right)$.

differences between the predictions is most noticeable in the positive peaks, but even there predictions vary from each other by no more than 10 Pascals.

\section{A New Metric for Comparison}

The question of where to place the Kirchhoff surface, and the analogous question of how far out to perform the quadrupole integration, have a strong impact on the decision of which method is most efficient computationally. Two things must be considered: i) the accuracy and extent of the CFD computation needed as input data for noise prediction, and ii) the amount of input data required. Both of these will depend on the size of the nonlinear region surrounding the body generating the noise. While at present we are unable to give a completely satisfactory answer to the above questions, we can provide some guidelines using a new metric as follows.

We note that the solution of the $\mathrm{FW}-\mathrm{H}$ equation with the quadrupole source term invariably involves $T_{i j}$ and its first and second derivatives. Therefore, it is imperative that not only $T_{i j}$ is calculated accurately, but also its first and second derivatives in the source region. Similarly, the Kirchhoff formula tells us that on the Kirchhoff surface, $p^{\prime}, \dot{p}^{\prime}$, and $\partial p^{\prime} / \partial n \equiv p_{n}^{\prime}$ must be computed accurately in the CFD solution. This indicates that the error analysis in all high resolution CFD computations must be based on the Sobolev norm. This norm is used very often in finite element analysis $^{22}$ and we propose such a norm in aeroacoustics. We will not present any numerical results in this paper based on the Sobolev norm.

We first address the problem of how to compare two high resolution CFD solutions for both $\mathrm{FW}-\mathrm{H}$ and
Kirchhoff methods. We assume all parameters of CFD and acoustic calculations are dimensionless. Let $V$ be the volume where CFD computations are performed with the boundary $\partial V$. We define the Sobolev norm of $T_{i j}$ as

$$
\begin{aligned}
\left\|T_{i j}\right\|_{V}= & \left\{\int _ { 0 } ^ { T } \int _ { V } \left[\sum_{i, j}\left|T_{i j}\right|^{2}+\sum_{j}\left|\frac{\partial T_{i j}}{\partial x_{i}}\right|^{2}\right.\right. \\
& \left.\left.+\left|\frac{\partial^{2} T_{i j}}{\partial x_{i} \partial x_{j}}\right|^{2}\right] d \mathbf{x} d t\right\}^{1 / 2}
\end{aligned}
$$

where $T$ is a convenient time period usually taken as the inverse of the blade passage frequency. We have now a metric for comparing two CFD calculations as follows. We define the distance (error) between two results by

$$
d\left(T_{i j}^{1}, T_{i j}^{2}\right)=\left\|T_{i j}^{1}-T_{i j}^{2}\right\|_{V}
$$

where $T_{i j}^{1}$ and $T_{i j}^{2}$ pertain to the two sets of computational results. We may agree that the error is small if

$$
\frac{\left\|T_{i j}^{1}-T_{i j}^{2}\right\|_{V}}{\left\|T_{i j}^{1}\right\|_{V}} \ll 1 .
$$

The two sets of results may come from two different CFD computations.

Now we consider the Kirchhoff method. Assume $S$ is the Kirchhoff surface over which the nondimensional $p^{\prime}, \dot{p}^{\prime}$, and $p_{n}^{\prime}$ are specified. We define a Sobolev norm of $p^{\prime}$ and distance for two solutions from CFD compu- 
tations $p^{1}$ and $p^{2}$ as follows:

$$
\begin{gathered}
\left\|p^{\prime}\right\|_{S}=\left\{\int_{0}^{T} \int_{S}\left[\left|p^{\prime}\right|^{2}+\left|\dot{p}^{\prime}\right|^{2}+\left|p_{n}^{\prime}\right|^{2}\right] d S d t\right\}^{1 / 2} \\
d\left(p^{\prime 1}, p^{\prime 2}\right)=\left\|p^{1}-p^{\prime 2}\right\|_{S} .
\end{gathered}
$$

We can use this distance or error function to know when to stop a CFD grid refinement. Unfortunately, this norm would not tell us when we are in the linear region or whether the dispersion and dissipation errors have substantially influenced $p^{\prime}, \dot{p}^{\prime}$, and $p_{n}^{\prime}$. These effects are governed by grid size as well as artificial viscosity. In the study of these effects in high resolution CFD calculations, we must employ a Sobolev norm in defining the computational errors.

An alternate use of the norm defined in equation (19) is to decide the volume of quadrupole source included in our noise calculations. Let $V_{1}$ and $V_{2}$ be two volumes such that $V_{1} \subset V_{2}$. Then assume that $T_{i j}^{1}=0$ outside $V_{1}$. Using the Sobolev norm with volume integration over $V_{2}$, we can say that $V_{1}$ includes all quadrupoles needed for noise calculation if

$$
\frac{\left\|T_{i j}^{1}-T_{i j}^{2}\right\|_{V_{2}}}{\left\|T_{i j}^{1}\right\|_{V_{1}}} \ll 1 .
$$

This means that

$$
\left\|T_{i j}^{2}\right\|_{V_{2} \backslash V_{1}} \ll\left\|T_{i j}^{1}\right\|_{V_{1}}
$$

where $V_{2} \backslash V_{1}$ is the volume enclosed between $\partial V_{1}$ and $\partial V_{2}$. This answers how far from the blade surface we must include quadrupole sources.

\section{Conclusions}

In this paper we have compared two useful aeroacoustic tools: i) the Lighthill acoustic analogy as embodied in the FW-H equation, and ii) the Kirchhoff formulation for moving surfaces. Both of these methodologies have proven their usefulness in rotor noise prediction. It is because both methods work well that it is difficult to determine which to use for a particular application. In a comparison of the governing equations, we have shown that the $\mathrm{FW}-\mathrm{H}$ approach can include nonlinear flow effects in the surface integration if the usual assumption of an impenetrable surface is relaxed. In fact, when the integration surface for each is in the linear flow region we have shown that the FW-H equation is equivalent to the Kirchhoff governing equation.

The FW-H equation is based on the conservation laws of fluid mechanics rather than the wave equation, as is the case for the Kirchhoff formula. As a consequence, the $\mathrm{FW}-\mathrm{H}$ equation is not appropriate for all types of wave propagation (e.g., the $\mathrm{FW}-\mathrm{H}$ equation is not appropriate for electromagnetic wave propagation, while the Kirchhoff formula could be utilized). But the superiority of the $\mathrm{FW}-\mathrm{H}$ for the aeroacoustics of rotating blades has been demonstrated through several numerical examples in this paper. The placement of the integration surface is a matter of convenience as long as the quadrupole source is utilized. The $\mathrm{FW}-\mathrm{H}$ method also has the advantage that it separates the predicted noise into physical components (i.e., thickness, loading, and quadrupole), explicitly. The Kirchhoff method does not offer this insight into the nature of the acoustic field.

It is well known that the quadrupole sources are responsible for noise generation as well as distortion of the acoustic waveform. The intense quadrupole sources are in the vicinity of the blades. Therefore, if we use a surface which encloses the blade and the volume of intense quadrupoles in the $\mathrm{FW}-\mathrm{H}$ method, we can calculate the level of the acoustic pressure accurately. The role of the weaker quadrupoles, which are farther away from the physical body, is primarily to provide a small distortion to the acoustic waveform. Hence, even when the integration surface is fairly close to the noise generating surface, it may be acceptable to neglect the external quadrupoles. In comparison, the Kirchhoff formula can predict acoustic pressures that are substantially in error if the Kirchhoff surface is located inside the nonlinear region. The nature and order of magnitude of this error may be hard to estimate or even recognize.

\section{References}

1. Lighthill, M. J., "On Sound Generated Aerodynamically, I: General Theory," Proceedings of the Royal Society, Vol. A221, 1952, pp. 564-587.

2. Ffowcs Williams, J. E., and Hawkings, D. L., "Sound Generated by Turbulence and Surfaces in Arbitrary Motion," Philosophical Transactions of the Royal Society, Vol. A264, No. 1151, 1969, pp. 321-342.

3. Farassat, F., and Myers, M. K., "Extension of Kirchhoff's Formula to Radiation from Moving Surfaces," Journal of Sound and Vibration, Vol. 123, No. 3, 1988, pp. 451-461.

4. Farassat, F., and Myers, M. K., "The Kirchhoff formula for a Supersonically Moving Surface," Proceedings of the 1st Joint CEAS / AIAA Aeroacoustics Conference (16th AIAA Aeroacoustics Conference), Vol. I, 1995, pp. 455-463. CEAS/AIAA Paper 95-062. 
5. Brentner, K. S., Lyrintzis, A. S., and Koutsavdis, E. K., "A Comparison of Computational Aeroacoustic Prediction Methods for Transonic Rotor Noise Prediction," American Helicopter Society 52nd Annual Forum, 1996.

6. Brentner, K. S., "Prediction of Helicopter Discrete Frequency Rotor Noise-A Computer Program Incorporating Realistic Blade Motions and Advanced Formulation," NASA TM 87721, Oct. 1986.

7. Brentner, K. S., and Holland, P. C., "An Efficient and Robust Method for Computing Quadrupole Noise," American Helicopter Society 2nd International Aeromechanics Specialists' Conference, Oct. 1995.

8. Brentner, K. S., "An Efficient and Robust Method for Predicting Helicopter Rotor High-Speed Impulsive Noise," AIAA Paper 96-0151, 1996.

9. Xue, Y., and Lyrintzis, A. S., "Rotating Kirchhoff Method for Three-Dimensional Transonic BladeVortex Interaction Hover Noise," AIAA Journal, Vol. 32, No. 7, July 1994, pp. 1350-1359.

10. Lyrintzis, A. S., Koutsavdis, E. K., Berezin, C., Visintainer, J., and Pollack, M., "Kirchhoff Acoustic Methodology Validation and Implementation in the TiltRotor Aeroacoustic Codes (TRAC)," American Helicopter Society 2nd International Aeromechanics Specialists' Conference, Oct. 1995.

11. Farassat, F., "Quadrupole Source in Prediction of Noise of Rotating Blades-A New Source Description," AIAA Paper 87-2675, 1987.

12. Farassat, F., and Brentner, K. S., "The Uses and Abuses of the Acoustic Analogy in Helicopter Rotor Noise Prediction," Journal of the American Helicopter Society, Vol. 33, 1988, pp. 29-36.

13. Farassat, F., and Myers, M. K., "An Analysis of the Quadrupole Noise Source of High Speed Rotating Blades," Computational AcousticsScattering, Gaussian Beams, and Aeroacoustics, edited by D. Lee, A. Cakmak, and R. Vichnevetsky, North-Holland, Amsterdam, Vol. 2, 1990, pp. 227-240.

14. di Francesantonio, P., "A New Kirchhoff Formulation for Transonic Rotor Noise," Twenty-Second European Rotorcraft Forum, Sept. 1996. Paper 83.

15. Pilon, A. R., and Lyrintzis, A. S., "Integral Methods for Computational Aeroacoustics," AIAA Paper 97-0020, Jan. 1997.
16. Farassat, F., and Succi, G. P., "The Prediction of Helicopter Discrete Frequency Noise," Vertica, Vol. 7, No. 4, 1983, pp. 309-320.

17. Baeder, J. D., Gallman, J. M., and Yu, Y. H., "A Computational Study of Aeroacoustics of Rotors in Hover," Journal of the American Helicopter Society, Vol. 42, No. 1, Jan. 1997, pp. 39-53.

18. Burley, C. L., and Tadghighi, H., "Importance of High Accuracy Blade Motion and Airloads Predictions in Acoustic Analysis," American Helicopter Society 50th Annual Forum, 1994.

19. Prichard, D. S., D. Douglas Boyd, J., and Burley, C. L., "NASA Langley's CFD-Based BVI Rotor Noise Prediction System: (ROTONET/FPRBVI) An Introduction and User's Guide," NASA TM 109147, Nov. 1994.

20. Purcell, T. W., "CFD and Transonic Helicopter Sound," Fourteenth European Rotorcraft Forum, 1988. Paper 2.

21. Schmitz, F. H., Boxwell, D. A., Splettstoesser, W. R., and Schultz, K. J., "Model-Rotor HighSpeed Impulsive Noise: Full-Scale Comparisons and Parametric Variations," Vertica, Vol. 8, No. 4, 1984, pp. 395-422.

22. Brenner, S. C., and Scott, C. R., The Mathematical Theory of Finite Element Methods, SpringerVerlag, 1994. 